\title{
BMJ Open How do people of South Asian origin understand and experience depression? A protocol for a systematic review of qualitative literature
}

\author{
Roisin Mooney, ${ }^{1}$ Daksha Trivedi, ${ }^{2}$ Shivani Sharma ${ }^{1}$
}

To cite: Mooney R, Trivedi D, Sharma S. How do people of South Asian origin understand and experience depression? A protocol for a systematic review of qualitative literature. BMJ Open 2016;6:e011697. doi:10.1136/bmjopen-2016011697

- Prepublication history for this paper is available online. To view these files please visit the journal online (http://dx.doi.org/10.1136/ bmjopen-2016-011697).

Received 26 February 2016 Revised 7 April 2016 Accepted 13 May 2016

CrossMark

\footnotetext{
${ }^{1}$ Department of Psychology and Sports Sciences, University of Hertfordshire, College Lane, Hatfield, Hertfordshire, UK ${ }^{2}$ Centre for Research in Primary and Community Care, University of Hertfordshire, Hatfield, Hertfordshire, UK
}

Correspondence to Dr Shivani Sharma; s.3.sharma@herts.ac.uk

\section{ABSTRACT}

Introduction: Individuals from Black and Asian Minority Ethnic (BAME) groups are less likely to receive a diagnosis and to engage with treatment for depression. This review aims to draw on international literature to summarise what is known about how people specifically of South Asian origin, migrants and non-migrants, understand and experience depressive symptoms. The resulting evidence base will further inform practices aimed at encouraging help-seeking behaviour and treatment uptake.

Methods and analysis: A systematic review and thematic synthesis of qualitative literature conducted according to the Preferred Reporting Items for Systematic reviews and Meta-Analyses (PRISMA) guidelines. Using predefined inclusion and exclusion criteria, electronic searches will be conducted across 16 databases. Study quality will be assessed using the Critical Appraisal Skills Programme (CASP). Data will be extracted independently by 2 reviewers.

Ethics and dissemination: Ethical approval is not required. A comprehensive evidence base of how people from South Asian backgrounds conceptualise and experience depression will better inform the design and delivery of mental health initiatives and advance directions for future research. Findings will be published in a peer-reviewed journal, and disseminated through existing networks for professionals, researchers, patients and the public.

Trial registration number: CRD42015026120.

\section{INTRODUCTION}

Depression is ranked as a leading cause of disability affecting around 350 million people worldwide. ${ }^{12}$ It has a profound effect on the lives of individuals, characterised by a range of cognitive, behavioural and somatic symptoms. ${ }^{3}$ The negative impact of depression on quality of life, as well as associated risk of mortality, is well documented, ${ }^{45}$ highlighting the need for effective screening, diagnosis and treatment.

Existing data suggest that the prevalence of common mental disorders (CMD), such as

\section{Strengths and limitations of this study}

- This will be the first comprehensive systematic review of how people of South Asian origin understand and experience depression.

- This review will result in an evidence base that can be drawn on by mental health professionals and researchers working in multiethnic settings.

- Analysis will only include literature that has been published in English due to the complexities involved in the translation and synthesis of data from multiple languages.

- The synthesis will rely mainly on data from primary research papers.

depression, is higher among people from Black and Asian Minority Ethnic (BAME) groups. ${ }^{6}$ For example, a recent study focusing on older adults ${ }^{8}$ reported that those from British South Asian and Black Caribbean backgrounds were considerably more likely to report depressive symptoms when compared with their white European counterparts. It is important to further understand the reasons for such disparities in mental health experiences and to encourage engagement with intervention. Improving uptake of mental health services by ethnic minorities is a longstanding challenge for public health providers ${ }^{9}$ in countries such as the UK where $\sim 14 \%$ of the population is identified as BAME, with the most prominent group being those with origins in South Asia (eg, India, Pakistan and Bangladesh). ${ }^{10}$ There are clear barriers to the take-up of mental health services among BAME groups with low engagement rates reported across numerous studies. ${ }^{11-13}$

In a focus group study with South Asian service users, ${ }^{14}$ it was determined that cultural exclusion was perceived throughout psychiatric services, which left patients feeling unable to share their concerns. The tendency for mental health services to be 
structured around western models of mental illness may in itself act as a barrier to the accurate identification and treatment of depressive symptoms. People often hold culture-specific beliefs about the causality of such conditions, which impacts not only symptom experiences, but also disposition to disclose the same. ${ }^{6}{ }^{15}$ Studies comparing people from white British and South Asian backgrounds in the UK have demonstrated higher rates of depressive symptoms among South Asians. ${ }^{7} 16$ However, it is also true that people of South Asian origin were more likely to disclose somatic rather than psychological symptoms. ${ }^{16}$ This is important since the expression of psychological issues in a somatic form may cloud the ability of professionals to attribute the symptoms to depressive mood over other illnesses. Those working in mental healthcare and in multiethnic contexts may therefore benefit from a comprehensive understanding of the beliefs that people from different ethnic backgrounds hold about depression including what it is, and its causes, alongside learning about symptom profiles from the experiences of those who have been affected by the condition. While there have been systematic reviews conducted in 2004 and 2007 concerning components of how South Asian people experience depression, ${ }^{16}{ }^{17}$ both of these reviews focused on research conducted with South Asian women. A more comprehensive evidence synthesis would help mental health professionals and policymakers in understanding the range of barriers and facilitators to providing effective care services, alongside informing researchers about future priorities to advance knowledge in the area.

In some cultures, there is no word for 'depression' per say, but the syndrome is thought to be synonymous with other more accepted terms. ${ }^{18} 19$ Direct translations are either non-existent or so scarcely used that they are poorly understood. Given that language barriers are also common, it would be useful to know about how different South Asian subgroups conceptualise depression and the unique or shared ways in which it is expressed. A comprehensive evidence synthesis would therefore be a helpful next step towards the development of mental healthcare practices for dominant BAME groups in the UK context. The nature of the questions that the review seeks to address necessitates a qualitative evidence synthesis to provide the level of richness in beliefs and experiences that may advance the most benefit for people contending with depressive symptoms.

This systematic review will be the first to undertake a comprehensive synthesis of the international qualitative literature on how people of South Asian origin understand and conceptualise depression alongside summarising the lived experiences of those who have been affected by the condition. The review will be purposefully broad in its focus on international literature since this will allow for a compassion of how, if at all, the beliefs and experiences of migrants differ from those of individuals based in South Asian regions. In doing so, we will address two primary questions:
- How do people of South Asian origin understand depression? This will include their unique descriptions of the syndrome alongside casual beliefs.

- How do people of South Asian origin experience symptoms of depression?

\section{METHODS AND DESIGN}

This protocol was developed using the Preferred Reporting Items for Systematic Reviews and Meta-Analyses (PRISMA-P) guidelines ${ }^{20}$ and has been registered on the PROSPERO database.

\section{Eligibility criteria \\ Types of studies}

Published and unpublished literature will be searched. Studies will be considered for inclusion if they utilise qualitative methods, are reported in English and were conducted after January 1990. We recognise that there may be research published in South Asian languages that will be highly relevant to the current review. However, the linguistic resources required to enable the inclusion of this literature is beyond the scope of the present research.

\section{Participants}

Studies conducted in Bangladesh, India, Nepal, Pakistan and Sri Lanka will be included alongside other localities with the caveat that participants identify themselves as South Asian. Only studies conducted with adults (aged 18 and over) will be considered.

\section{Settings}

There will be no restriction on the setting. This is to allow exploration of lay beliefs, alongside capturing the diversity of patient experiences that can be gleaned from studies based in community mental health and other long-term illness settings.

\section{Outcomes}

The main outcomes will be how people understand and experience depression, as gleaned from qualitative research findings. This will include data concerning the causes attributed to and how depression is defined by members of the South Asian population. Additionally, we are interested in barriers and facilitators to the disclosure of symptoms and engagement with treatment. We will extract relevant data and develop themes supported by verbatim quotes.

\section{Identification of studies}

Qualitative studies may be difficult to identify ${ }^{21}$ owing to a lack of established methodological filters and MeSH headings. Furthermore, issues pertaining to depression may be embedded in research with a different focus, for example, studies into mental health experiences in the context of long-term health conditions. Our search strategy will be designed to be broad and sensitive enough to 
ensure that all potentially relevant studies are captured. Lateral searching techniques will also be employed, which have been shown to be important for identifying non-randomised studies. ${ }^{22}$ Searches will include:

- Electronic databases including: MEDLINE (PubMed), Scopus, PsycArticles, CINAHL, ResearchGate, King's Fund database, the Cochrane Library (including Cochrane Central Register of Controlled Trials (CENTRAL), CDSR, DARE, HTA) and NTIS;

- Checking of reference lists from primary studies and systematic reviews (snowballing) $;^{23}$

- Citation searches using the 'Cited by' option on Google Scholar and Scopus, and the 'Related articles' option on PubMed ('Lateral Searching') ${ }^{22}$

- Contact with experts and those with an interest in this field to uncover grey literature (eg, South Asian Community Mental Health Service (AMARDEEP Lambeth UK), MIND, WHO);

- Ongoing studies on the NIHR Portfolio and hand searching highly relevant journals.

\section{Search strategy}

There are three aspects to our search strategy. The first relates to the population. Preliminary searches will help uncover the breadth of terminology used to reference people from South Asian backgrounds, as the definition of this population and its subgroups varies as a function of where research has been conducted. For example, the term Indo-Asian is more prominent in some parts of the world, whereas South Asian is the most widely used term within the UK. Additionally, we will include countries of origin in order to further encompass relevant literature.

Second, in relation to depression, it is well established that in many South Asian languages, there is no direct equivalent for the term. As such, we will search for related concepts such as distress, mood and mental wellbeing. We will use MeSH and free text terms and our lateral searching methods will help identify relevant literature including those where the experience of depression is embedded among other issues. Finally, searches will be limited to qualitative literature.

\section{Study screening and selection}

Electronic search results will be imported into Endnote bibliographic software, and any duplicates deleted. Two reviewers will independently screen titles and abstracts against the inclusion criteria. Full manuscripts of potentially relevant literature will be obtained and screened by two researchers using a screening form with clearly defined criteria. Disagreements will be resolved by discussion with a third reviewer, and where necessary, primary authors of the manuscripts will be contacted for further information.

\section{Quality assessment}

Included studies will be appraised using the Critical Appraisal Skills Programme (CASP) checklist for qualitative studies, which has been used in previous reviews. ${ }^{23-25}$ This consists of a series of questions within the following domains; appropriateness of the research design, recruitment strategy, data collection, position of the researcher, ethics, rigour of the analysis, clarity of findings and value of the research. Two reviewers will use the checklist independently and then meet to agree the final quality score. Discrepancies will be resolved by discussion or by consultation with the third author. We will include all studies regardless of their quality as there is no consensus or empirically tested method for excluding qualitative studies from reviews on this basis. However, we will undertake sensitivity analyses by exploring the relative contribution made by studies to the findings according to study quality. Such an approach has been recommended by other researchers, ${ }^{26}$ and will help to further assess the impact of study quality on review outcomes.

\section{Data extraction}

Two reviewers will independently extract data into a predesigned and piloted spreadsheet under the following headings; Study title, Author, Year, Type of article (Research/Review/Position paper), Study design, Study aims/research questions, Data collection methods (eg, Interview, Focus groups), Methods of data analysis (eg, Grounded Theory, Thematic Analysis, Interpretative Phenomenological Analysis), Types of participants (eg, Age, sex, ethnicity, socio-economic status, existing co-morbidities), Settings (eg, Country, location, community or hospital), Outcomes, Language (eg, What language was the data collected and analysed in?).

\section{Data analysis and synthesis}

We will use thematic analysis to synthesise study findings to determine key concepts across the included studies based on methods employed in previous systematic reviews. ${ }^{26}{ }^{27}$ This will involve three stages: the coding of text, the development of descriptive themes and the generation of analytic themes. Determining what 'data' should be considered for analysis can be challenging. ${ }^{26}$ Therefore, 'data' will include quotations as well as sections labelled as 'findings' or 'results'. Included studies will be independently reviewed and coded by two reviewers. Codes will be used to identify themes that have emerged from the papers and will be refined by constant reference to the primary data source and discussion, documenting quotes as supporting evidence. ${ }^{27}$ Associations between the different themes will be identified and grouped into higher-order themes, which will be used to answer the review questions. Further analysis of qualitative data will be conducted using the NVivo software (V.10.2.1), which enables reviewers to see which data from studies or subpopulations have contributed to each of the themes ${ }^{26}$ assisting the development of representative and accurate themes. The results of this synthesis will be reported according to the Enhancing transparency in reporting the synthesis of qualitative research: ENTREQ, statement. ${ }^{28}$ 


\section{DISCUSSION AND DISSEMINATION}

Engaging people from BAME groups in mental health services is a challenge, despite a recognised need for support in tackling CMD such as depression. ${ }^{15}$ Those working in multiethnic contexts would benefit from the most accurate knowledge on factors that may impact disparities in healthcare uptake. This review will advance a comprehensive evidence synthesis relating to people of South Asian origin who represent the most dominant BAME group in the UK. ${ }^{10}$ The main outcomes will be an enhanced understanding of how people of South Asian origin experience depression, and clarity on how they define depression as a construct. Such knowledge will include information surrounding the beliefs held by this population and potentially its subgroups, such as migrants versus those based in South Asia, concerning the aetiology of depression and relevant symptoms. The findings will potentially benefit mental health practitioners and policymakers by providing an evidence base that could be used to inform service design and delivery. For example, helping to inform mental health awareness raising campaigns by targeting them at the information needs of specific groups or tailoring them in a way that is culturally sensitive. The findings will also be of use to researchers in the field in setting future research priorities. For example, by encompassing the international literature, the review may help inform the debate as to whether depression is a culture-specific or culture general construct and so how it should be measured. ${ }^{29-31}$

Twitter Follow Roisin Mooney at @Roisin_MM

Contributors SS conceived the study concept. DT provided expertise on the systematic review methodology. RM drafted the protocol manuscript. All authors provided feedback throughout the development of the protocol and approved the final manuscript.

Funding This review will be undertaken as a component of a $\mathrm{PhD}$ at the University of Hertfordshire.

Competing interests None declared.

Provenance and peer review Not commissioned; externally peer reviewed.

Open Access This is an Open Access article distributed in accordance with the Creative Commons Attribution Non Commercial (CC BY-NC 4.0) license, which permits others to distribute, remix, adapt, build upon this work noncommercially, and license their derivative works on different terms, provided the original work is properly cited and the use is non-commercial. See: http:// creativecommons.org/licenses/by-nc/4.0/

\section{REFERENCES}

1. Ferrari AJ, Charlson FJ, Norman RE, et al. Burden of depressive disorders by country, sex, age, and year: findings from the global burden of disease study 2010. PLOS Med 2013;10:e1001547.

2. Marcus M, Yasamy MT, van Ommeren M, et al. Depression: a global public health concern. In: Health WFfM, ed. Depression: a global crisis. Geneva (Switzerland): WHO, 2012.

3. Simon GE, VonKorff M, Piccinelli M, et al. An international study of the relation between somatic symptoms and depression. $N$ Engl J Med 1999;341:1329-35.

4. Moussavi S, Chatterji S, Verdes E, et al. Depression, chronic diseases, and decrements in health: results from the World Health Surveys. Lancet 2007:370:851-8.

5. Sullivan PF, Neale MC, Kendler KS. Genetic epidemiology of major depression: review and meta-analysis. Am J Psychiatry 2000;157:1552-62.
6. Williams DR, González HM, Neighbors $\mathrm{H}$, et al. Prevalence and distribution of major depressive disorder in African Americans, Caribbean blacks, and non-hispanic whites: results from The National survey of American life. Arch Gen Psychiatry 2007:64:305-15.

7. Rees R, Stokes G, Stansfield C, et al. Prevalence of mental health disorders in adult minority ethnic populations in England: a systematic review. London: EPPI-Centre Social Science Research Unit, UCL Institute of Education, University College London, 2016.

8. Williams ED, Tillin T, Richards $M$, et al. Depressive symptoms are doubled in older British South Asian and Black Caribbean people compared with Europeans: associations with excess co-morbidity and socioeconomic disadvantage. Psychol Med 2015;45:1861-71.

9. Bhui K, Stansfeld S, Hull S, et al. Ethnic variations in pathways to and use of specialist mental health services in the UK. Systematic review. Br J Psychiatry 2003;182:105-16.

10. ONS. Ethnicity and national identity in England and Wales 2011. London, UK: Office for National Statistics, 2012;1-15.

11. Williams DR, Mohammed SA. Discrimination and racial disparities in health: evidence and needed research. J Behav Med 2009:32:20-47.

12. Ikram UZ, Snijder MB, Fassaert TJL, et al. The contribution of perceived ethnic discrimination to the prevalence of depression. Eur J Public Health 2015;25:243-8.

13. Gary FA. STIGMA: barrier to mental health care among ethnic minorities. Issues Ment Health Nurs 2005;26:979-99.

14. Bowl R. The need for change in UK mental health services: South Asian service users' views. Ethn Health 2007:12:1-19.

15. Weich S, Nazroo J, Sproston K, et al. Common mental disorders and ethnicity in England: the EMPIRIC Study. Psychol Med 2004;34:1543.

16. Hussain F, Cochrane R. Depression in South Asian women living in the UK: a review of the literature with implications for service provision. Transcult Psychiatry 2004;41:253-70.

17. Trivedi JK, Mishra M, Kendurkar A. Depression among women in the South-Asian region: the underlying issues. J Affect Disord 2007;102:219-25.

18. Parker G, Gladstone G, Chee KT. Depression in the planet's largest ethnic group: the Chinese. Am J Psychiatry 2001;158:857-64.

19. Bhugra D, Mastrogianni A. Globalisation and mental disorders. Br J Psychiatry 2003;184:10-20.

20. Shamseer L, Moher D, Clarke M, et al. Preferred reporting items for systematic review and meta-analysis protocols (PRISMA-P) 2015: elaboration and explanation. BMJ 2015;349:g7647.

21. Wong SS, Wilczynski NL, Haynes RB. Developing optimal search strategies for detecting clinically relevant qualitative studies in MEDLINE. Stud Health Technol Inform 2004;107:311-16.

22. Greenhalgh T, Peacock R. Effectiveness and efficiency of search methods in systematic reviews of complex evidence: audit of primary sources. BMJ 2005;331:1064-5.

23. Dixon-Woods M, Sutton A, Shaw R, et al. Appraising qualitative research for inclusion in systematic reviews: a quantitative and qualitative comparison of three methods. J Health Serv Res Policy 2007;12:42-7.

24. Hawker S, Payne S, Kerr C, et al. Appraising the evidence: reviewing disparate data systematically. Qual Health Res 2002;12:1284-99.

25. Walter FM, Emery J, Braithwaite D, et al. Lay understanding of familial risk of common chronic diseases: a systematic review and synthesis of qualitative research. Ann Fam Med 2004;2:583-94.

26. Thomas J, Harden A. Methods for the thematic synthesis of qualitative research in systematic reviews. BMC Med Res Methodol 2008;8:45.

27. Bunn F, Goodman C, Sworn K, et al. Psychosocial factors that shape patient and carer experiences of dementia diagnosis and treatment: a systematic review of qualitative studies. PLoS Med 2012;9:e1001331.

28. Tong A, Flemming K, Mclnnes E, et al. Enhancing transparency in reporting the synthesis of qualitative research: ENTREQ. BMC Med Res Methodol 2012;12:181.

29. Kuo WH. Prevalence of depression among Asian-Americans. J Nerv Ment Dis 1984;172:449-57.

30. Lloyd CE, Roy T, Begum S, et al. Measuring psychologica well-being in South Asians with diabetes; a qualitative investigation of the PHQ-9 and the WHO-5 as potential screening tools for measuring symptoms of depression. Diabet Med 2012:29:140-7.

31. Sharma S, Bhui K, Chilcot J, et al. Identifying depression in South asian patients with end-stage renal disease: considerations for practice. Nephron Extra 2011;1:262-71. 